https://helda.helsinki.fi

Genetics and genomics in postoperative pain and analgesia

\author{
Palada, Vinko
}

2018-10

Palada , V , Kaunisto , M A \& Kalso , E 2018 , ' Genetics and genomics in postoperative pain and analgesia ' , Current opinion in anaesthesiology , vol. 31 , no. 5 , pp. 569-574 . https://doi.org/10.1097/ACO.000

http://hdl.handle.net/10138/308801

https://doi.org/10.1097/ACO.0000000000000633

publishedVersion

Downloaded from Helda, University of Helsinki institutional repository.

This is an electronic reprint of the original article.

This reprint may differ from the original in pagination and typographic detail.

Please cite the original version. 


\title{
Genetics and genomics in postoperative pain and analgesia
}

\author{
Vinko Palada ${ }^{\mathrm{a}}$, Mari A. Kaunisto ${ }^{\mathrm{b}}$, and Eija Kalso ${ }^{\mathrm{a}, \mathrm{c}}$
}

\begin{abstract}
Purpose of review
The review describes recent advances in genetics and genomics of postoperative pain, the association between genetic variants and the efficacy of analgesics, and the role of pharmacogenomics in the selection of appropriate analgesic treatments for postoperative pain.
\end{abstract}

\section{Recent findings}

Recent genetic studies have reported associations of genetic variants in catechol-O-methyltransferase (COMT), brain-derived neurotrophic factor (BDNF), voltage-gated channel alpha subunit 11 (SCN11A) and $\mu$-opioid receptor (OPRM1) genes with postoperative pain. The recent pharmacogenetics studies revealed an association of the organic cation transporter 1 (OCT1) and ATP-binding cassette C3 (ABCC3) polymorphisms with morphine-related adverse effects, an effect of polymorphisms in cytochrome P450 gene CYP2D6 on the analgesic efficacy of tramadol and no effect of CYP2C8 and CYP2C9 variants on efficacy of piroxicam.

\begin{abstract}
Summary
Genetic variants associate with inter-individual variability in drug responses and they can affect pain sensitivity and intensity of postoperative pain. Despite the recent progress in genetics and genomics of postoperative pain, it is still not possible to precisely predict the patients who are genetically predisposed to have severe postoperative pain or who develop chronic postoperative pain.
\end{abstract}

\section{Keywords}

analgesics, genetic variants, genetics, pharmacogenetics, postoperative pain

\section{INTRODUCTION}

Postoperative pain is a major health burden due to increasing prevalence and inadequate efficacy of current treatments [1-4]. All patients undergoing surgery have acute postoperative pain which usually resolves within days to weeks during wound healing. Some patients, however, develop chronic postoperative pain (CPOP), which is usually defined as pain due to surgery lasting for more than 3 months. The severity of acute postoperative pain correlates with the risk of CPOP [5]. Its incidence varies depending on the type of surgery, criteria for pain and duration of follow-up. Fletcher et al. [6] have reported an incidence of moderate to severe CPOP of $11.8 \%$ at 1 year after surgery whereas Haroutiunian et al. [7] reported the incidence of $6-68 \%$ for postoperative neuropathic pain depending on the type of surgery. Several risk factors are associated with the development of CPOP such as preoperative pain, type of surgery, perioperative nerve injury, fear of surgery, pain catastrophizing, anxiety, expectation of pain, obesity, young age and sex (female) [8-11].
Single nucleotide polymorphisms (SNPs) of several pain regulating genes have been reported to associate with increased pain sensitivity and higher risk for postoperative pain [12-14].

Pharmacogenetics refers to genetic factors that associate with variation in drug response. The most studied pharmacogenetic effects on analgesics are related to the mutations in the $\mu$-opioid receptor gene (OPRM1) [15]. Individuals who are homozygous for the minor allele of the OPRM1 rs1799971 $(118 \mathrm{~A}>\mathrm{G})$ have been reported to need about 30\% more oxycodone to achieve adequate analgesia

\footnotetext{
${ }^{a}$ Department of Physiology and Pharmacology, Karolinska Institutet, Stockholm, Sweden, ${ }^{b}$ Institute for Molecular Medicine Finland (FIMM), HiLIFE, University of Helsinki, Helsinki, Finland and ${ }^{\mathrm{C} P a i n}$ Clinic, Department of Anaesthesiology, Intensive Care and Pain Medicine, Helsinki University Hospital and University of Helsinki, Finland
}

Correspondence to Eija Kalso, MD, PhD, Pain Clinic, PB 140, 00029 HUS, Finland. Tel: +358 9 47175885; e-mail: eija.kalso@helsinki.fi

Curr Opin Anesthesiol 2018, 31:569-574

DOI:10.1097/ACO.0000000000000633 


\section{KEY POINTS}

- Candidate gene studies have identified genetic variants in several genes that might predispose patients to more severe postoperative pain.

- Genetic variants can modulate the severity of postoperative pain, analgesic efficacy, metabolism, and adverse events of analgesics.

- Pharmacogenetics can improve pain management by predicting the individual response to analgesics prior to therapy and facilitate the development of novel and more effective pain medication for postoperative pain.

- Larger cohorts using standardized analgesia methods are needed for future progress in this field.

compared with the individuals who are homozygous for the major allele [16]. Drugs metabolized by the CYP2D6 isoenzyme are particularly liable for significant variation in efficacy between individuals as the CYP2D6 gene is highly polymorphic [17]. Codeine is an excellent example. It is a pro-drug that needs to be metabolized to morphine for analgesic efficacy. Due to variation in the activity of CYP2D6, individuals can produce morphine very efficiently (ultrarapid metabolizers) or not at all (poor metabolizers) [18]. Pharmacogenetics may play a role in many other functions relevant to pharmacokinetics, for example in the transporters [19].

In this review, we will discuss the recent advances in genetics and genomics of postoperative pain, the association between genetic variants and the efficacy of analgesics and the important role of pharmacogenomics in the selection of appropriate analgesic treatments for postoperative pain.

\section{GENETICS OF POSTOPERATIVE PAIN}

Advances in genome-wide association studies (GWAS) have helped to identify the hereditary factors that might predispose patients to postoperative pain [20-22]. Despite the advantage of GWAS studies that are able to genotype thousands of common genetic variants $(>1 \%$ frequency in population) across the genome [14], the large majority of genetic studies on postoperative pain are still candidate gene studies focusing on individual gene polymorphisms. Emerging next generation sequencing (NGS) technologies enable identification of both common and rare genetic variants [23] but are still to be used for screening of genetic variants in postoperative pain. Recent genetic studies have focused on investigating the association of genetic variants in a limited set of functionally relevant genes with postoperative pain. Variants in catechol-O-methyltransferase (COMT), brain-derived neurotrophic factor $(B D N F)$, voltage-gated channel alpha subunit 11 $(S C N 11 A)$ and $\mu$-opioid receptor (OPRM1) genes have shown evidence of association with postoperative pain.

Mladenovic et al. [24"] investigated the association between COMT gene polymorphisms and temporomandibular disorders (TMD), TMD pain and postoperative pain after third molar surgery. The COMT SNPs rs6269, rs4633, rs4818 and rs4680 were associated with pain sensitivity and a higher risk to develop TMD which has high incidence in young adults after third molar extraction $[25,26]$. Targeted genotyping of blood samples from 90 patients with painful TMD and 92 controls was performed for the COMT SNPs rs6269, rs4680 and rs165774 revealing an association of AA and AG genotypes with rs165774 (G>A, intron) polymorphism and increased risk of TMD compared with the GG genotype. In addition, the AA genotype for rs6269 polymorphism (A>G, 5'UTR) was associated with lower postoperative TMD pain and acute pain at the dental extraction site. These findings suggest that chronic postoperative TMD pain and acute pain at the extraction site after third moral surgery may be associated with COMT rs6269 polymorphism.

Khalil et al. [27"] studied the potential effect of interaction between COMT SNPs (rs6269, rs4633, rs4818, and rs4680) with OPRM1 rs1799971 polymorphism on postoperative pain and opioid consumption in 153 patients after orthopaedic surgery. It was reported that genetic variants of COMT can modulate the OPRM1 gene expression and density in the brain by regulating the level of enkephalin $[28,29]$. A significant interaction was observed for OPRM1 and the low pain sensitivity (LPS) haplotype of COMT. In patients without the LPS haplotype, carriers of the AA genotype of OPRM1 were associated with significantly higher postoperative pain scores compared with carriers of the $G$ allele. Patients carrying Met158Met of the COMT rs4680 and $\mathrm{AG} / \mathrm{GG}$ of the OPRM1 or TT of the COMT rs 4633 and the AG/GG of OPRM1 had the highest amount of opioid consumption. These results suggest that interactions of OPRM1 and COMT might contribute to postoperative pain and response to opioids.

Tian et al. [30"'] analysed the association of 638 polymorphisms in 54 pain-related genes with $\mathrm{CPOP}$ in 1152 Chinese surgical patients. The patients carrying the $\mathrm{G}$ allele (Val/Met and $\mathrm{Val} / \mathrm{Val}$ genotypes) for SNP rs6265 (Val66Met) in BDNF gene were associated with a higher risk of CPOP compared with the carriers of wild type A allele (Met/Met genotype). The $B D N F^{\text {Met/Met }}$ mice were associated with lower mechanical allodynia compared with $B D N F^{\mathrm{Val} / \mathrm{Val}}$ 
mice after plantar incision. These results indicate that BDNF rs6265 polymorphism may be associated with an increased risk of CPOP.

Sun et al. [31"] examined the association of SNPs in the SCN11A gene with postoperative pain sensitivity in 309 Han Chinese female patients after gynaecological laparoscopic surgery. In total, 5 SNPs (rs33985936, rs13080116, rs11720988, rs11709492, and rs11720013) in SCN11A were associated with experimental pain sensitivity and minor alleles of rs33985936 and rs13080116 were linked with increased postoperative patient-controlled analgesia consumption of sufentanil and tramadol (13.2\% increased PCA for rs33985936 C/T compared to C/C genotype). This finding suggests that SCN11A SNPs may associate with postoperative pain after gynaecological surgery.

Werner et al. [32] performed a GWAS analysis of neuropathic pain in 613 patients with osteoarthritis (OA) after total joint replacement. The detected variants did not pass the genome-wide significance $P$-value threshold $\left(5 \times 10^{-8}\right)$. The strongest association was found for rs887797 variant in protein kinase $\mathrm{C}$ alpha (PRKCA) previously associated with inflammatory pain [33].

Persson et al. [34] studied the association of polymorphisms in 13 candidate pain genes $(A B C B 1$, COMT, PEBP1, CYP2D6, OPRM1, CYP34A, POMC, MAOB, SCN9A, UGT2B7, SUDS3, TAOK3, VSIG10) with pain sensitivity after laparoscopic cholecystectomy. Due to the low number of patients $(n=57)$, there were no statistically significant differences after multiple variant analyses based on high and low intensity pain phenotypes for more than 400 SNPs in 13 candidate genes.

\section{PHARMACOGENETICS OF POSTOPERATIVE PAIN}

Pharmacogenetic studies investigate the effect of genetic variants on the efficacy, metabolism and adverse effects of drugs, which might result in heterogeneous individual responses to drugs [35]. As long-term opioid administration after surgery can lead to opioid-induced hyperalgesia [36], pharmacogenetics might improve pain management by predicting the individual response to analgesics prior to the therapy and could help to develop more effective analgesics. Recent pharmacogenetics studies investigated the association of organic cation transporter 1 (OCT1) and ATP-binding cassette C3 (ABCC3) SNPs with morphine-related adverse effects as well as the effect of polymorphisms in cytochrome P450 genes CYP2C8, CYP2C9 and $C Y P 2 D 6$ on the analgesic efficacy of tramadol and piroxicam.
Balyan et al. [37"'] studied whether the functional polymorphisms in the OCT1 gene are associated to postoperative morphine-related adverse effects including respiratory depression (RD) and postoperative nausea and vomiting (PONV) in 311 children undergoing tonsillectomy. The OCT1 transporter mediates hepatic uptake of cationic drugs including morphine [38]. It was found that the OCT1 variant rs12208357 was associated with PONV whereas SNP rs72552763 was associated with RD. Previous studies have linked rs12208357 to decreased OCT1 protein expression [39] and rs72552763 to the increased plasma concentrations of tropisetron, ondansetron and tramadol [40]. This study suggests that genetic variants leading to decreased OCT1 transporter activity and higher morphine plasma levels are associated with a higher incidence of morphine-related RD and PONV.

Chidambaran et al. [41"'] studied the association between SNPs in the ATP-binding cassette ABCC3 and morphine-induced $\mathrm{RD}$ and pharmacokinetics (PK) of morphine in two independent cohorts of children who underwent tonsillectomy $(n=316)$ or adolescents after spine surgery $(n=67)$. The $A B C C 3$ gene encodes for the liver transporter which plays an important role in morphine metabolism through glucuronidation to morphine-3-glucuronide (M3G) and morphine-6-glucuronide (M6G) [42]. Gene-specific analysis of genome-wide genotype data identified 7 intronic SNPs (rs35364174, rs4148412, rs739923, rs733392, rs1978153, rs7216383, rs886493) in the $A B C C 3$ gene which were significantly associated with prolonged stay in the postoperative anaesthesia care unit (PACU) due to morphine-induced RD. Furthermore, ABCC3 rs4793665 CC genotype was associated with higher clearance of M3G and M6G compared with CT and TT genotypes in both tonsillectomy and spine surgery subjects. This study suggests that $A B C C 3$ variants are associated with postoperative $\mathrm{RD}$, prolonged hospital stay and morphine $\mathrm{PK}$, and that children genetically predisposed for higher risk of morphine-related RD could be preoperatively identified in order to use alternative analgesics that are not transported through ABCC3 transporters.

Stamer et al. [43"'] analysed the effect of loss-offunction polymorphism in the organic cation transporter OCT1 and variants in CYP2D6 on the pharmacokinetics and analgesic efficacy of the opioid analgesic tramadol in 205 patients after abdominal or urological surgery. Genetic variants in CYP2D6 and OCT1 modulate the pharmacokinetics and analgesic efficacy of tramadol $[38,44]$. Multiple regression analysis identified that the number of active OCT1 alleles and CYP2D6 had a significant impact on tramadol consumption. Furthermore, the loss of 
function SNPs in the OCT1 gene were associated with reduced tramadol consumption and increased plasma concentrations of the active tramadol metabolite (1)O-desmethyltramadol in the patients. These results suggest the importance of genotypedependent pharmacogenetic dosing of tramadol based on OCT1 and CYP2D6 activity.

Bialecka et al. [45] addressed the effect of interleukin 6 (IL-6) functional polymorphism rs1800795 $(\mathrm{G}>\mathrm{C})$ on opioid requirements in OA patients after total hip replacement (THR). Healthy $\mathrm{C}$ allele carriers are known to have decreased $I L-6$ gene expression and reduced plasma levels of IL- 6 compared with the G allele carriers [46] which might be associated with milder inflammation and lower nociceptive stimulation. By genotyping 196 patients after THR for the IL-6 rs1800795 SNP, it was observed that patients with GG and GC genotypes had significantly increased administration of opioids on days 0,3 and 4 after THR compared to patients with CC genotype. This indicates that G allele and higher IL-6 levels may predispose OA patients to increased opioid requirements in the early postoperative phase after THR.

Calvo et al. $\left[47^{\circ}\right.$ investigated the association between polymorphisms in CYP2C8 and CYP2C9 genes with the efficacy of the nonsteroidal antiinflammatory drug (NSAID) piroxicam during the management of postoperative pain following lower third molar surgery. The elimination half-life of piroxicam is about $45 \mathrm{~h}$ [48]. The carriers of the CYP2C9 mutant allele 3 (1/3 and $3 / 3$ genotypes) were previously reported to have slower metabolism of piroxicam compared with people homozygous for the wild type allele 1 (CYP2C91/1 genotype) [49] while some SNPs of $C Y P 2 C 8$ such as $C Y P 2 C 8 * 3$ were reported to decrease the metabolism of NSAIDs [50]. Based on genotyping of 105 volunteers, no differences were found in efficacy of piroxicam and adverse effects between the carriers of normal and slow metabolizing $C Y P 2 C 8 * 3$ and $C Y P 2 C 9$ alleles after 4 days administrations. These results indicate that piroxicam regulates the inflammation after third molar surgery without influence from CYP genotypes.

Somogyi et al. [51"] studied the contribution of ethnicity-dependent genetic variability in innate immune signalling to morphine consumption during PCA in 598 Han Chinese, 230 Malay and 133 Indian women after elective caesarean section under spinal anaesthesia. The incidence of postoperative pain was significantly higher in Chinese patients with COMT rs4680 genotypes which was not observed in Malay and Indian cohorts. Interaction between ethnicity and OPRM1 variant rs1799971, Toll-like receptor 2 (TLR2) variant rs3804100 and
IL-6 variant rs1143634 predicted $9.8 \%$ of the variability in morphine consumption in the entire cohort. These findings indicate that innate immune system might contribute to the variability in postoperative morphine requirements in an ethnicitydependent manner.

Senagore et al. [52"'] analysed the effect of pharmacogenetics-guided selection of analgesics following major abdominal surgery within an enhanced recovery protocol (ERP). The analgesic protocol was guided based on the assessment of CYP1A2, CYP2C19, CYP2C9, CYP2D6, CYP3A4, CYP3A5, COMT, OPRM1 and ABCB1 genes which were part of a diagnostic pain gene panel. The evaluation was performed for 63 patients undergoing open or laparoscopic colorectal and major ventral hernia surgeries and 47 control patients undergoing the same operations while being managed with standard ERP. Pharmacogenomic guidance resulted in modifications of opioid analgesia of the ERP in $80 \%$ of the patients and changes in the choice of NSAID in 56\% of the patients. These changes resulted in a 50\% reduction in opioid consumption and reduced adverse effects compared with standard ERP.

Yuan et al. [53] investigated the effect of the pregnane $\mathrm{X}$ receptor $P X R * 1 B$ polymorphisms on CYP3A4 enzyme activity and postoperative consumption of the $\mu$-opioid receptor agonist fentanyl in 287 Han Chinese female patients undergoing abdominal total hysterectomy or myomectomy. PXR is a nuclear receptor which regulates the expression of CYP3A4 [54], the liver enzyme responsible for the metabolism of fentanyl [55]. There were no significant differences in the consumption of fentanyl $24 \mathrm{~h}$ after surgery, pain scores and the enzyme activity of CYP3A4 in Chinese female patients based on the $P X R * 1 B$ haplotypes which might be due to the low number of patients $(n=95)$ with complete clinical data and information regarding CYP3A enzyme activity.

\section{CONCLUSION}

In general, the main predictive factor for postoperative pain is type of surgery [56]. Other major factors include preoperative pain, anxiety and age $[8,56]$. On an individual basis, genetic variation may play a significant role in pain intensity and treatment outcome. However, despite the recent progress in genetics and genomics of postoperative pain, it is still not possible to predict how much pain a patient will have postoperatively. Future genetic studies should consider recruiting larger patient cohorts with standardized analgesic protocols in order to achieve sufficient statistical power to detect genetic variants associated with postoperative pain. Also, 
more advanced methods such as NGS should be utilized to screen for rare genetic variants that can modulate pain. Furthermore, it is encouraged to perform meta-analyses of previous studies and to independently reproduce the findings in different research groups. Utilizing the novel genome-editing technologies such as CRISPR/Cas9 should help to develop new animal models for postoperative pain which can better model the development of postoperative pain in humans. The associations between epigenetic changes and postoperative pain are poorly understood and require more intensive research. The emerging diagnostic pain panels can be used to select more effective analgesic treatments based on genotype-dependent dosing of analgesics. Finally, pharmacogenetics might facilitate the development of novel analgesics for postoperative pain with less adverse effects by taking into account the inter-individual variability in drug responses.

\section{Acknowledgements}

None.

\section{Financial support and sponsorship}

None.

\section{Conflicts of interest}

There are no conflicts of interest.

\section{REFERENCES AND RECOMMENDED \\ READING}

Papers of particular interest, published within the annual period of review, have been highlighted as:

- of special interest

m. of outstanding interest

1. Macrae WA. Chronic pain after surgery. Br J Anaesth $2001 ; 87: 88-98$.

2. Rawal N. Postoperative pain treatment for ambulatory surgery. Best Pract Res Clin Anaesthesiol 2007; 21:129-148.

3. Johannes CB, Le TK, Zhou X, et al. The prevalence of chronic pain in United States adults: results of an internet-based survey. J Pain 2010; 11: 1230-1239.

4. Parsons $B$, Schaefer $C$, Mann R, et al. Economic and humanistic burden of posttrauma and postsurgical neuropathic pain among adults in the United States. J Pain Res 2013; 6:459-469.

5. Katz J, Jackson M, Kavanagh BP, et al. Acute pain after thoracic surgery predicts long-term postthoracotomy pain. Clin J Pain 1996; 12:50-55.

6. Fletcher D, Stamer UM, Pogatzki-Zahn E, et al. Chronic postsurgical pain in Europe: an observational study. Eur J Anaesthesiol 2015; 32:725-734.

7. Haroutiunian S, Nikolajsen L, Finnerup NB, et al. The neuropathic component in persistent postsurgical pain: a systematic literature review. Pain 2013; 154:95-102.

8. Kehlet $\mathrm{H}$, Jensen TS, Woolf $\mathrm{CJ}$. Persistent postsurgical pain: risk factors and prevention. Lancet 2006; 367:1618-1625.

9. Ip HY, Abrishami A, Peng PW, et al. Predictors of postoperative pain and analgesic consumption: a qualitative systematic review. Anesthesiol 2009; 111:657-677.

10. Katz J, Selzer Z. Transition from acute to chronic postsurgical pain: risk factors and protective factors. Expert Rev Neurother 2009; 9:723-744.

11. Sipilä RM, Haasio L, Meretoja TJ, et al. Does expecting more pain make it more intense? Factors associated with the first week pain trajectories after breast cancer surgery. Pain 2017; 158:922-930.

12. Sorge RE, Trang $T$, Dorfman $R$, et al. Genetically determined $P 2 X 7$ receptor pore formation regulates variability in chronic pain sensitivity. Nat Med 2012; 18:595-599.
13. Kambur O, Kaunisto MA, Tikkanen E, et al. Effect of catechol-o-methyltransferase-gene (COMT) variants on experimental and acute postoperative pain in 1,000 women undergoing surgery for breast cancer. Anesthesiol 2013; 119:1422-1433.

14. Clarke $\mathrm{H}$, Katz J, Flor $\mathrm{H}$, et al. Genetics of chronic postsurgical pain: a crucial step toward personal pain medicine. Can J Anesth 2015; 62:294-303.

15. Hwang IC, Park JY, Myung SK, et al. OPRM1 A118G gene variant and postoperative opioid requirement: a systematic review and meta-analysis. Anesthesiol 2014; 121:825-834.

16. Cajanus K, Holmström EJ, Wessman $M$, et al. Effect of endocannabinoid degradation on pain: role of FAAH polymorphisms in experimental and postoperative pain in women treated for breast cancer. Pain 2016; 157: $361-369$.

17. Hicks JK, Swen JJ, Gaedigk A. Challenges in CYP2D6 phenotype assignment from genotype data: a critical assessment and call for standardization. Curr Drug Metab 2014; 15:218-232.

18. Kirchheiner J, Schmidt $H$, Tzvetkov $M$, et al. Pharmacokinetics of codeine and its metabolite morphine in ultra-rapid metabolizers due to CYP2D6 duplication. Pharmacogenomics J 2007; 7:257-265.

19. Franke RM, Gardner ER, Sparreboom A. Pharmacogenetics of drug transporters. Curr Pharm Des 2010; 16:220-230.

20. Kim H, Ramsay $\mathrm{E}$, Lee $\mathrm{H}$, et al. Genome-wide association study of acute postsurgical pain in humans. Pharmacogenomics 2009; 10:171-179.

21. Nishizawa D, Fukuda K, Kasai $S$, et al. Genome-wide association study identifies a potent locus associated with human opioid sensitivity. Mol Psychiatry 2014; 19:55-62.

22. Mieda $T$, Nishizawa D, Nakagawa $H$, et al. Genome-wide association study identifies candidate loci associated with postoperative fentanyl requirements after laparoscopic-assisted colectomy. Pharmacogenomics 2016; 17:133-145.

23. Koboldt DC, Meltz Steinberg $K$, Larson $D E$, et al. The next-generation sequencing revolution and its impact on genomics. Cell 2013; 155:27-38.

24. Mladenovic I, Supic G, Kozomara R, et al. Genetic polymorphisms of catechol-

- O-methyltransferase: association with temporomandibular disorders and postoperative pain. J Oral Facial Pain Headache 2016; 30:302-310.

Study shows that chronic postoperative TMD pain and acute pain at the extraction site after third moral surgery may be associated with COMT rs6269 polymorphism

25. Diatchenko L, Slade GD, Nackley AG, et al. Genetic basis for individual variations in pain perception and the development of a chronic pain condition. Hum Mol Genet 2005; 14:135-143.

26. Huang GJ, Rue TC. Third-molar extraction as a risk factor for temporomandibular disorder. J Am Dent Assoc 2006; 137:1547-1554.

27. Khalil $H$, Sereika SM, Dai F, et al. OPRM1 and COMT gene-gene interaction is associated with postoperative pain and opioid consumption after orthopedic trauma. Biol Res Nurs 2017; 19:170-179.

Novel interaction of OPRM1 and COMT might contribute to postoperative pain and response to opioids.

28. Zubieta JK, Heitzeg MM, Smith $Y R$, et al. COMT val158met genotype affects mu-opioid neurotransmitter responses to a pain stressor. Science 2003; 299:1240-1243.

29. Berthele A, Platzer S, Jochim B, et al. COMT Val108/158Met genotype affects the mu-opioid receptor system in the human brain: evidence from ligand-binding, G-protein activation and preproenkephalin mRNA expression. Neuroimage 2005; 28:185-193.

30. Tian Y, Liu X, Jia M, et al. Targeted genotyping identifies susceptibility locus in

n. brain-derived neurotrophic factor gene for chronic postsurgical pain. Anesthesiology 2018; 128:587-597.

The BDNF rs6265 polymorphism may be associated with an increased risk of chronic postoperative pain.

31. Sun J, Duan G, Li N, et al. SCN11A variants may influence postoperative pain

- sensitivity after gynecological surgery in Chinese Han female patients. Medicine (Baltimore) 2017; 96:e8149.

The SCN11A SNPs may associate with postoperative pain after gynecological surgery.

32. Warner SC, van Meurs JB, Schiphof D, et al. Genome-wide association scan of neuropathic pain symptoms post total joint replacement highlights a variant in the protein-kinase C gene. Eur J Hum Genet 2017; 25:446-451.

33. Olah Z, Karai L, ladarola MJ. Protein kinase $C$ (alpha) is required for vanilloid receptor 1 activation. Evidence for multiple signaling pathways. J Biol Chem 2002; 277:35752-35759

34. Persson AKM, Pettersson FD, Åkeson J. Single nucleotide polymorphisms associated with pain sensitivity after laparoscopic cholecystectomy. Pain Med 2017; 19:1271-1279.

35. Ting $S$, Schug $S$. The pharmacogenomics of pain management: prospects for personalized medicine. J Pain Res 2016; 9:49-56.

36. Koppert W, Schmelz M. The impact of opioid-induced hyperalgesia for postoperative pain. Best Pract Res Clin Anaesthesiol 2007; 21:65-83.

37. Balyan $\mathrm{R}$, Zhang $\mathrm{X}$, Chidambaran $\mathrm{V}$, et al. OCT1 genetic variants are

n. associated with postoperative morphine-related adverse effects in children. Pharmacogenomics 2017; 18:621-629.

The genetic variants leading to decreased OCT1 transporter activity and higher morphine plasma levels are associated with the higher incidence of morphinerelated respiratory depression and postoperative nausea and vomiting in children. 
38. Tzvetkov MV, Saadatmand AR, Lotsch J, et al. Genetically polymorphic OCT1: another piece in the puzzle of the variable pharmacokinetics and pharmacodynamics of the opioidergic drug tramadol. Clin Pharmacol Ther 2011; 90:143-150.

39. Nies AT, Koepsell $\mathrm{H}$, Winter $\mathrm{S}$, et al. Expression of organic cation transporters OCT1 (SLC22A1) and OCT3 (SLC22A3) is affected by genetic factors and cholestasis in human liver. Hepatology 2009; 50:1227-1240.

40. Tzvetkov MV, Dos Santos Pereira JN, Meineke l, et al. Morphine is a substrate of the organic cation transporter OCT1 and polymorphisms in OCT1 gene affect morphine pharmacokinetics after codeine administration. Biochem Pharmacol 2013; 86:666-678.

41. Chidambaran $V$, Venkatasubramanian $R$, Zhang $X$, et al. ABCC3 genetic

- variants are associated with postoperative morphine-induced respiratory depression and morphine pharmacokinetics in children. Pharmacogenomics J 2017; 17:162-169.

This study identified $\mathrm{ABCC} 3$ variants associated with postoperative respiratory depression, prolonged hospital stay and morphine pharmacokinetics and suggests that children genetically predisposed for higher risk of morphine related respiratory depression could be preoperatively identified in order to use alternative analgesics that are not transported through $\mathrm{ABCC} 3$ transporters.

42. Thorn CF, Klein TE, Altman RB. Codeine and morphine pathway. Pharmacogenet Genomics 2009; 19:556-558.

43. Stamer UM, Musshoff F, Stüber F, et al. Loss-of-function polymorphisms in the

-1. organic cation transporter OCT1 are associated with reduced postoperative tramadol consumption. Pain 2016; 157:2467-2475

Novel insight into importance of genotype-dependent pharmacogenetic dosing of tramadol based on OCT1 and CYP2D6 activity.

44. Stamer UM, Lehnen K, Höthker F, et al. Impact of CYP2D6 genotype on postoperative tramadol analgesia. Pain 2003; 105:231-238.

45. Białecka $M$, Jurewicz $A$, Machoy-Mokrzyńska $A$, et al. Effect of interleukin 6-174G $>C$ gene polymorphism on opioid requirements after total hip replacement. J Anesth 2016; 30:562-567.

46. Fishman D, Faulds $G$, Jeffery $R$, et al. The effect of novel polymorphisms in the interleukin-6 (IL-6) gene on IL-6 transcription and plasma IL-6 levels, and an association with systemic-onset juvenile chronic arthritis. J Clin Invest 1998; 102:1369-1376.
47. Calvo AM, Zupelari-Gonçalves $P$, Dionísio TJ, et al. Efficacy of piroxicam for

- postoperative pain after lower third molar surgery associated with CYP $2 \mathrm{C} 8 * 3$ and CYP2C9. J Pain Res 2017; 10:1581-1589.

Piroxicam regulates the inflammation after third molar surgery without the influence from CYP genotypes.

48. Hobbs DC. Pharmacokinetics of piroxicam in man. Eur J Rheumatol Inflamm $1983 ; 6: 46-55$.

49. Perini JA, Vianna-Jorge R, Brogliato AR, et al. Influence of CYP2C9 genotypes on the pharmacokinetics and pharmacodynamics of piroxicam. Clin Pharmacol Ther 2005; 78:362-369.

50. Lopez-Rodriguez R, Novalbos J, Gallego-Sandin S, et al. Influence of CYP2C8 and CYP2C9 polymorphisms on pharmacokinetic and pharmacodynamic parameters of racemic and enantiomeric forms of ibuprofen in healthy volunteers. Pharmacol Res 2008; 58:77-84.

51. Somogyi AA, Sia AT, Tan EC, et al. Ethnicity-dependent influence of innate

- immune genetic markers on morphine PCA requirements and adverse effects in postoperative pain. Pain 2016; 157:2458-2466.

Innate immune system might contribute to the variability in postoperative morphine requirements in an ethnicity-dependent manner.

52. Senagore AJ, Champagne BJ, Dosokey E, et al. Pharmacogenetics-guided

- analgesics in major abdominal surgery: Further benefits within an enhanced recovery protocol. Am J Surg 2017; 213:467-472.

Pharmacogenomic selection of analgesics can reduce the opioid consumption and adverse effects compared to standard enhanced recovery protocol.

53. Yuan JJ, Ma XJ, Li ZS, et al. Effect of Pregnane X Receptor*1B genetic polymorphisms on postoperative analgesia with fentanyl in Chinese patients undergoing gynecological surgery. BMC Med Genet 2016; 17:87.

54. Smutny $T$, Mani S, Pavek P. Posttranslational and posttranscriptional modifications of pregnane $X$ receptor (PXR) in regulation of the cytochrome P450 superfamily. Curr Drug Metab 2013; 14:1059-1069.

55. Tateishi T, Krivoruk $Y$, Ueng YF, et al. Identification of human liver cytochrome $\mathrm{P}-4503 \mathrm{~A} 4$ as the enzyme responsible for fentanyl and sufentanil $\mathrm{N}$-dealkylation. Anesth Analg 1996; 82:167-172.

56. Cajanus $\mathrm{K}$, Neuvonen $\mathrm{M}$, Koskela $\mathrm{O}$, et al. Analgesic plasma concentrations of oxycodone after surgery for breast cancer-which factors matter? Clin Pharmacol Ther 2018; 103:653-662. 